\title{
A $q$-analogue of the matrix sixth Painlevé system
}

\author{
Hiroshi KAWAKAMI* \\ College of Science and Engineering, Aoyama gakuin university, 5-10-1 Fuchinobe, Chuo-ku, \\ Sagamihara-shi, Kanagawa 252-5258, Japan.
}

\begin{abstract}
We derive a $q$-analogue of the matrix sixth Painlevé system via a connection-preserving deformation of a certain Fuchsian linear $q$-difference system. In specifying the linear $q$-difference system, we utilize the correspondence between linear differential systems and linear $q$-difference systems from the viewpoint of the spectral type. The system of non-linear $q$-difference equations thus obtained can also be regarded as a non-abelian analogue of Jimbo-Sakai's $q-P_{\mathrm{VI}}$.
\end{abstract}

Mathematics Subject Classifications (2010). 34M55, 34M56, 33E17

Key words. q-difference equation, connection-preserving deformation, isomonodromic deformation, Painlevé-type equation, integrable system.

\section{Introduction}

The Painlevé equations are non-linear second order ordinary differential equations that define new special functions. Originally, the Painlevé equations were classified into six equations. We denote them by $P_{\mathrm{I}}, \ldots, P_{\mathrm{VI}}$. Among the Painlevé equations, the sixth Painlevé equation $P_{\mathrm{VI}}$ is the "source" in the sense that all the other Painlevé equations can be obtained from $P_{\mathrm{VI}}$ through degenerations.

Since the 1990s, there have been many generalizations of the Painlevé equations in the literature such as discretizations, higher dimensional analogues, quantizations, and so on. As for second order (or two-dimensional) discrete Painlevé equations, there is an algebro-geometric theory [13] which provides a unified account of them. According to the theory, they fall into three types of equations, namely, additive difference, multiplicative difference ( $q$-difference), and elliptic difference equations. The Painlevé (differential) equations are understood through the continuous limit of those discrete Painlevé equations. In this sense we can say that discrete Painlevé equations are more fundamental than the Painlevé differential equations.

Recently, Painlevé-type differential equations with four-dimensional phase space are classified from the viewpoint of isomonodromic deformations of linear differential equations [5, 8, 9, 10, This

${ }^{*}$ kawakami@gem.aoyama.ac.jp 
study shows that, in the four-dimensional case, there are four "sources" as extensions of the sixth Painlevé equation. Namely, they are

- the Garnier system [4], which is a classically known multivariate extension of $P_{\mathrm{VI}}$,

- the Fuji-Suzuki-Tsuda system [3, 19], which is an extension of $P_{\mathrm{VI}}$ with the affine Weyl group symmetry of type $A$,

- the Sasano system [16], which is an extension of $P_{\mathrm{VI}}$ with the affine Weyl group symmetry of type $D$,

- the matrix sixth Painlevé system [1, 5, which is a non-abelian extension of $P_{\mathrm{VI}}$.

Note that each of the four equations has its extensions defined in arbitrary even dimensions. These four families are expected to play an important role in the theories of integrable systems, special functions and so on.

Our next purpose is, motivated by the two-dimensional case, to develop a unified framework of discrete Painlevé-type equations in higher dimensions. As a first step, we investigate a correspondence of Painlevé-type differential equations and discrete Painlevé-type equations.

Among the above four families, $q$-analogues of the Garnier system, the Fuji-Suzuki-Tsuda system, and the Sasano system have already been constructed and studied by several authors [14, 17, 18, 11. The aim of this paper is to obtain a $q$-analogue of the matrix sixth Painlevé system (abbreviated to matrix $P_{\mathrm{VI}}$ ).

The matrix $P_{\mathrm{VI}}$ is derived from an isomonodromic deformation of a certain Fuchsian differential equation. The linear equation is specified in terms of the spectral type. The spectral type of a Fuchsian differential equation is data on the multiplicities of the characteristic exponents of a Fuchsian differential equation [12] (see Section 2). On the other hand, the notion of spectral type is also defined for Fuchsian linear $q$-difference equations [15]. Thus we have the spectral type for both Fuchsian differential and $q$-difference equations.

The author observed the relationship between the two spectral type (see Conjecture 3.3), and this correspondence is expected to be applicable to a construction of a $q$-analogue of a given isomonodromic deformation equation. That is, suppose we are given an isomonodromic deformation equation of a Fuchsian differential equation. Specify a linear $q$-difference equation which corresponds to the Fuchsian differential equation in terms of the spectral type. Then consider the connection-preserving deformation of the linear $q$-difference equation. The connection-preserving deformation [6] is a discrete counterpart of the isomonodromic deformation of linear differential equations. This paper provides an example of the construction.

This paper is organized as follows. In Section 2, we review the matrix sixth Painlevé system. In Section 3, we explain the connection-preserving deformation of a linear $q$-difference system and the spectral type for Fuchsian $q$-difference systems. We also present a conjecture concerning a correspondence between the spectral type of $q$-difference systems and that of differential systems. In Section 4, we derive a $q$-analogue of the matrix $P_{\mathrm{VI}}$. In Section 5, we show that the resulting system of non-linear $q$-difference equations actually has a continuous limit to the matrix $P_{\mathrm{VI}}$. 


\section{The matrix sixth Painlevé system}

In this section we review the matrix $P_{\mathrm{VI}}$ [7, 10]. The matrix $P_{\mathrm{VI}}$ is derived from the isomonodromic deformation of a certain Fuchsian differential system. First we give a brief account of the isomonodromic deformation of Fuchsian systems.

Consider a system of Fuchsian differential equations:

$$
\frac{d Y}{d x}=\mathcal{A}(x, u) Y, \quad \mathcal{A}(x, u)=\sum_{j=1}^{n} \frac{\mathcal{A}_{j}}{x-u_{j}} \quad\left(\mathcal{A}_{j} \in M_{m}(\mathbb{C})\right) .
$$

We put $\mathcal{A}_{\infty}:=-\sum_{j=1}^{n} \mathcal{A}_{j}$. For simplicity, we assume that $\mathcal{A}_{j}$ 's $(j=1, \ldots, n, \infty)$ are semisimple and that $\mathcal{A}_{\infty}$ is diagonal. Positions of the singular points $u=\left(u_{1}, \ldots, u_{n}\right)$ are regarded as deformation parameters. It is known that the normalized local solution of (2.1) at $x=\infty$ is an isomonodromic solution (that is, associated monodromy matrices are independent of $u$ ) if and only if the following system is completely integrable:

$$
\left\{\begin{array}{l}
\frac{\partial Y}{\partial x}=\mathcal{A}(x, u) Y, \\
\frac{\partial Y}{\partial u_{i}}=\mathcal{B}_{i}(x, u) Y, \quad \mathcal{B}_{i}(x, u)=-\frac{\mathcal{A}_{i}}{x-u_{i}} \quad(i=1, \ldots, n) .
\end{array}\right.
$$

Then the compatibility condition of the above

$$
\left\{\begin{array}{l}
\frac{\partial \mathcal{A}(x, u)}{\partial u_{i}}-\frac{\partial \mathcal{B}_{i}(x, u)}{\partial x}+\left[\mathcal{A}(x, u), \mathcal{B}_{i}(x, u)\right]=O \\
\frac{\partial \mathcal{B}_{i}(x, u)}{\partial u_{j}}-\frac{\partial \mathcal{B}_{j}(x, u)}{\partial u_{i}}+\left[\mathcal{B}_{i}(x, u), \mathcal{B}_{j}(x, u)\right]=O
\end{array}\right.
$$

gives a system of non-linear differential equations satisfied by the entries of $\mathcal{A}_{i}$ 's. Here we put $[A, B]:=A B-B A$. We can write the compatibility condition more explicitly as follows:

$$
\left\{\begin{array}{l}
\frac{\partial A_{i}}{\partial u_{j}}=\frac{\left[A_{j}, A_{i}\right]}{u_{j}-u_{i}} \quad(j \neq i) \\
\frac{\partial A_{i}}{\partial u_{i}}=-\sum_{j \neq i} \frac{\left[A_{j}, A_{i}\right]}{u_{j}-u_{i}}
\end{array}\right.
$$

This system is the isomonodromic deformation equation of (2.1), which is called the Schlesinger system.

Fuchsian systems are classified by their spectral types. Let the eigenvalues of $\mathcal{A}_{j}$ be $\theta_{k}^{j}(k=$ $\left.1, \ldots, \ell_{j}\right)$, and let their multiplicities be $m_{k}^{j}\left(k=1, \ldots, \ell_{j}\right)$ respectively. Regarding $\mathcal{P}_{j}=m_{1}^{j}, \ldots, m_{\ell_{j}}^{j}$ as a partition of $m$, we have a $(n+1)$-tuple of partitions $\mathcal{P}_{1} ; \ldots ; \mathcal{P}_{n} ; \mathcal{P}_{\infty}$. We call this $(n+1)$-tuple of partitions of $m$ the spectral type of (2.1).

The matrix $P_{\mathrm{VI}}$ is derived from the isomonodromic deformation of the following Fuchsian system:

$$
\frac{d Y}{d x}=\mathcal{A}(x, t) Y, \quad \mathcal{A}(x, t)=\frac{\mathcal{A}_{0}}{x}+\frac{\mathcal{A}_{1}}{x-1}+\frac{\mathcal{A}_{t}}{x-t},
$$


where $\mathcal{A}_{0}, \mathcal{A}_{1}$, and $\mathcal{A}_{t}$ are $2 m \times 2 m$ matrices satisfying the following conditions

$$
\mathcal{A}_{0} \sim\left(\begin{array}{cc}
O_{m} & O_{m} \\
O_{m} & \theta^{0} I_{m}
\end{array}\right), \quad \mathcal{A}_{1} \sim\left(\begin{array}{cc}
O_{m} & O_{m} \\
O_{m} & \theta^{1} I_{m}
\end{array}\right), \quad \mathcal{A}_{t} \sim\left(\begin{array}{cc}
O_{m} & O_{m} \\
O_{m} & \theta^{t} I_{m}
\end{array}\right),
$$

and

$$
\mathcal{A}_{\infty}=-\left(\mathcal{A}_{0}+\mathcal{A}_{1}+\mathcal{A}_{t}\right)=\operatorname{diag}(\overbrace{\theta_{1}^{\infty}, \ldots, \theta_{1}^{\infty}}^{m}, \overbrace{\theta_{2}^{\infty}, \ldots, \theta_{2}^{\infty}}^{m-1}, \theta_{3}^{\infty}) .
$$

Thus the spectral type of the Fuchsian system (2.5) is $m, m ; m, m ; m, m ; m, m-1,1$ and the number of accessory parameters is $2 m$. Taking the trace of (2.7), we have the Fuchs relation

$$
m\left(\theta^{0}+\theta^{1}+\theta^{t}+\theta_{1}^{\infty}\right)+(m-1) \theta_{2}^{\infty}+\theta_{3}^{\infty}=0 .
$$

The linear systems satisfying the conditions (2.6) and (2.7) can be parametrized as follows ([7]):

$$
\begin{aligned}
& \mathcal{A}_{\xi}=\left(U \oplus I_{m}\right)^{-1} X^{-1} \hat{\mathcal{A}}_{\xi} X\left(U \oplus I_{m}\right) \quad(\xi=0,1, t), \\
& \hat{\mathcal{A}}_{0}=\left(\begin{array}{c}
I_{m} \\
O_{m}
\end{array}\right)\left(\begin{array}{ll}
\theta^{0} I_{m} & \frac{1}{t} Q-I_{m}
\end{array}\right), \quad \hat{\mathcal{A}}_{1}=\left(\begin{array}{c}
I_{m} \\
P Q-\Theta
\end{array}\right)\left(\begin{array}{ll}
\theta^{1} I_{m}-P Q+\Theta & I_{m}
\end{array}\right), \\
& \hat{\mathcal{A}}_{t}=\left(\begin{array}{c}
I_{m} \\
t P
\end{array}\right)\left(\begin{array}{ll}
\theta^{t} I_{m}+Q P & -\frac{1}{t} Q
\end{array}\right),
\end{aligned}
$$

where the matrix $\Theta$ is given by

$$
\Theta=\left(\begin{array}{cc}
\theta_{2}^{\infty} I_{m-1} & O \\
O & \theta_{3}^{\infty}
\end{array}\right)
$$

The matrices $Q$ and $P$ satisfy the following commutation relation

$$
[P, Q]=\left(\theta+\theta_{1}^{\infty}\right) I_{m}+\Theta .
$$

Here we put $\theta=\theta^{0}+\theta^{1}+\theta^{t}$. Hereafter we often write a scalar matrix $k I$ as $k$ if there is no danger of confusion. The matrix $U \in \mathrm{GL}_{m}(\mathbb{C})$ is a gauge variable, and $X$ is given by $X=\left(\begin{array}{cc}I_{m} & O \\ Z & I_{m}\end{array}\right)$ where

$$
Z=\left(\theta_{1}^{\infty}-\Theta\right)^{-1}\left[-\theta^{1}\left(Q P+\theta+\theta_{1}^{\infty}\right)+\left(Q P+\theta+\theta_{1}^{\infty}\right)^{2}-t\left(P Q+\theta^{t}\right) P\right]
$$

As mentioned in the above, the isomonodromic deformation of (2.5) is equivalent to the compatibility condition of the following Lax pair:

$$
\left\{\begin{array}{l}
\frac{\partial Y}{\partial x}=\mathcal{A}(x, t) Y \\
\frac{\partial Y}{\partial t}=\mathcal{B}(x, t) Y
\end{array}\right.
$$

where $\mathcal{B}(x, t)=-\frac{\mathcal{A}_{t}}{x-t}$. Then the compatibility condition

$$
\frac{\partial \mathcal{A}(x, t)}{\partial t}-\frac{\partial \mathcal{B}(x, t)}{\partial x}+[\mathcal{A}(x, t), \mathcal{B}(x, t)]=O
$$


of the Lax pair (2.13) can be explicitly written as

$$
\begin{aligned}
t(t-1) \frac{d Q}{d t}= & (Q-t) P Q(Q-1)+Q(Q-1) P(Q-t) \\
& +\left(\theta^{0}+1\right) Q(Q-1)+\left(\theta+2 \theta_{1}^{\infty}-1\right) Q(Q-t)+\theta^{t}(Q-1)(Q-t), \\
t(t-1) \frac{d P}{d t}= & -(Q-1) P(Q-t) P-P(Q-t) P Q-P Q(Q-1) P \\
& -\left[\left(\theta^{0}+1\right)\{P(Q-1)+Q P\}+\left(\theta+2 \theta_{1}^{\infty}-1\right)\{P(Q-t)+Q P\}+\theta^{t}\{P(Q-t)+(Q-1) P\}\right] \\
& -\left(\theta+\theta_{1}^{\infty}\right)\left(\theta^{0}+\theta^{t}+\theta_{1}^{\infty}\right), \\
t(t-1) \frac{d U}{d t}= & \left\{(Q-t)(P Q+Q P)+\left(2 \theta^{0}+\theta^{1}+2 \theta^{t}+2 \theta_{1}^{\infty}\right) Q-\theta^{t} t\right\} U .
\end{aligned}
$$

Moreover, equations (2.15) and (2.16) can be written in the following form:

$$
\frac{d q_{i j}}{d t}=\frac{\partial H_{\mathrm{VI}}^{\mathrm{Mat}, m}}{\partial p_{j i}}, \quad \frac{d p_{i j}}{d t}=-\frac{\partial H_{\mathrm{VI}}^{\mathrm{Mat}, m}}{\partial q_{j i}} \quad(i, j=1, \ldots, m)
$$

in terms of the entries of $Q=\left(q_{i j}\right)$ and $P=\left(p_{i j}\right)$. The Hamiltonian is given by

$$
\begin{aligned}
& t(t-1) H_{\mathrm{VI}}^{\mathrm{Mat}, m} \\
& =\operatorname{tr}\left[Q(Q-1)(Q-t) P^{2}+\left\{\left(\theta^{0}+1-\left(\theta+\theta_{1}^{\infty}+\Theta\right)\right) Q(Q-1)+\theta^{t}(Q-1)(Q-t)\right.\right. \\
& \left.\left.+\left(\theta+2 \theta_{1}^{\infty}-1\right) Q(Q-t)\right\} P+\left(\theta+\theta_{1}^{\infty}\right)\left(\theta^{0}+\theta^{t}+\theta_{1}^{\infty}\right) Q\right] .
\end{aligned}
$$

We call the equations (2.15) and (2.16), or equivalently the Hamiltonian system (2.18), the matrix sixth Painlevé system (matrix $\left.P_{\mathrm{VI}}\right)$. We construct a $q$-analogue of the equations (2.15) and (2.16) in Section 4 .

\section{Linear $q$-difference systems and their deformations}

In this section, we collect some facts about linear $q$-difference equations that will be used later.

\subsection{Connection-preserving deformation of linear $q$-difference systems}

Let $q$ be a complex number satisfying $0<|q|<1$. We consider a system of linear $q$-difference equations

$$
Y(q x)=A(x) Y(x),
$$

where $A(x)=A_{N} x^{N}+\cdots+A_{1} x+A_{0}$ is an $m \times m$ matrix with polynomial entries. We assume that the matrices $A_{N}$ and $A_{0}$ are semisimple and invertible. Using a gauge transformation $Y(x) \rightarrow$ $G Y(x)$ with a constant matrix $G$, we can assume that $A_{N}$ is diagonal without loss of generality. We set

$$
A_{0}=G_{0} \operatorname{diag}\left(\theta_{1}, \ldots, \theta_{m}\right) G_{0}^{-1}, \quad A_{N}=\operatorname{diag}\left(\kappa_{1}, \ldots, \kappa_{m}\right)
$$

and

$$
D_{0}=\frac{1}{\log q} \operatorname{diag}\left(\log \theta_{1}, \ldots, \log \theta_{m}\right), \quad D_{\infty}=\frac{1}{\log q} \operatorname{diag}\left(\log \kappa_{1}, \ldots, \log \kappa_{m}\right)
$$


We also assume the non-resonant condition, that is, for any $i, j$

$$
\theta_{j} / \theta_{i}, \kappa_{j} / \kappa_{i} \notin q^{\mathbb{Z}_{\geq 1}}=\left\{q^{n} \mid n \in \mathbb{Z}_{\geq 1}\right\}
$$

Under the above assumptions, it is known that the system (3.1) has the following solutions at $x=0$ and $x=\infty$ :

Proposition 3.1 ([2]). The system (3.1) has the following solutions

$$
\begin{aligned}
Y_{0}(x) & =G_{0} \hat{Y}_{0}(x) x^{D_{0}} \\
Y_{\infty}(x) & =q^{\frac{N}{2} u(u-1)} \hat{Y}_{\infty}(x) x^{D_{\infty}} \quad(u=\log x / \log q),
\end{aligned}
$$

where $\hat{Y}_{0}(x)$ (resp. $\hat{Y}_{\infty}(x)$ ) is a invertible matrix whose entries are holomorphic at $x=0$ (resp. $x=\infty)$ and satisfies $\hat{Y}_{0}(0)=I_{m}\left(\right.$ resp. $\left.\hat{Y}_{\infty}(\infty)=I_{m}\right)$.

By using (3.1), we have for any $k \in \mathbb{Z}_{\geq 1}$

$$
\begin{aligned}
\hat{Y}_{0}(x) & =G_{0}^{-1} A(x)^{-1} A(q x)^{-1} \cdots A\left(q^{k-1} x\right)^{-1} G_{0} \hat{Y}_{0}\left(q^{k} x\right) G_{0}^{-1} A_{0}^{k} G_{0}, \\
\hat{Y}_{\infty}(x)^{-1} & =q^{-\frac{k(k+1) N}{2}} x^{k N} A_{N}^{k} \hat{Y}_{\infty}\left(q^{-k} x\right)^{-1} A\left(q^{-k} x\right)^{-1} \cdots A\left(q^{-2} x\right)^{-1} A\left(q^{-1} x\right)^{-1} .
\end{aligned}
$$

These expressions show that $\hat{Y}_{0}(x)^{-1}$ and $\hat{Y}_{\infty}(x)$ can be analytically continued to $\mathbb{P}^{1} \backslash\{0, \infty\}$, while $\hat{Y}_{0}(x)$ and $\hat{Y}_{\infty}(x)^{-1}$ can be meromorphically continued to the same domain. Let $\alpha_{j}(j=1, \ldots, m N)$ be the zeros of $\operatorname{det} A(x)$. Then the matrix $\hat{Y}_{0}(x)$ may have poles at

$$
\left\{q^{-k} \alpha_{j} \mid k \in \mathbb{Z}_{\geq 0}, j=1, \ldots, m N\right\}
$$

and the matrix $\hat{Y}_{\infty}(x)^{-1}$ may have poles at

$$
\left\{q^{k} \alpha_{j} \mid k \in \mathbb{Z}_{\geq 1}, j=1, \ldots, m N\right\} .
$$

The connection matrix $P(x)$ is defined on $\mathbb{P}^{1} \backslash\{0, \infty\}$ as follows:

$$
Y_{\infty}(x)=Y_{0}(x) P(x)
$$

Obviously, $P(x)$ is pseudo-constant, that is, $P(q x)=P(x)$.

Next we consider a deformation of the system. We can choose some of eigenvalues $\theta_{i}$ 's, $\kappa_{j}$ 's, and zeros $\alpha_{j}$ 's of $\operatorname{det} A(x)$ as deformation parameters. For simplicity, we assume that the number of deformation parameters is one. Let us denote such a parameter by $t$. Then, in general, the connection matrix $P(x)$ depends on $t ; P=P(x, t)$.

The connection-preserving deformation requires that the connection matrix $P(x, t)$ be pseudoconstant with respect to the parameter $t$. The following holds:

Proposition 3.2. The connection matrix $P(x, t)$ is pseudo-constant with respect to $t$, namely $P(x, q t)=P(x, t)$, if and only if

$$
Y_{\infty}(x, q t) Y_{\infty}(x, t)^{-1}=Y_{0}(x, q t) Y_{0}(x, t)^{-1} .
$$


Proof. This is immediate from

$$
Y_{\infty}(x, q t) Y_{\infty}(x, t)^{-1}=Y_{0}(x, q t) P(x, q t) P(x, t)^{-1} Y_{0}(x, t)^{-1}
$$

We put $B(x, t):=Y_{\infty}(x, q t) Y_{\infty}(x, t)^{-1}=Y_{0}(x, q t) Y_{0}(x, t)^{-1}$. Then the solutions $Y(x, t)=$ $Y_{0}(x, t), Y_{\infty}(x, t)$ satisfy

$$
Y(x, q t)=B(x, t) Y(x, t) .
$$

Under some general conditions, the matrix $B(x, t)$ is shown to be rational in $x$. Conversely, if the solutions $Y(x, t)=Y_{0}(x, t), Y_{\infty}(x, t)$ satisfy (3.14) for some matrix $B(x, t)$ which is rational in $x$, then the corresponding connection matrix is pseudo-constant in $t$.

Thus (under some conditions) the connection-preserving deformation is equivalent to the existence of $B(x, t)$ which is rational in $x$ such that the following pair

$$
\left\{\begin{array}{l}
Y(q x, t)=A(x, t) Y(x, t) \\
Y(x, q t)=B(x, t) Y(x, t)
\end{array}\right.
$$

is compatible. Then, analogous to the isomonodromic deformation of linear differential equations, the compatibility condition

$$
A(x, q t) B(x, t)=B(q x, t) A(x, t)
$$

of (3.15) reduces to a system of non-linear $q$-difference equations satisfied by entries of $A(x, t)$ and $B(x, t)$. Actually, the entries of $B(x, t)$ can be expressed by entries of $A(x, t)$. In Section 4 , we see how to determine the matrix $B(x, t)$ through an example.

\subsection{Spectral types of linear $q$-difference systems}

Here we recall the notion of spectral type of linear $q$-difference systems introduced in [15]. For simplicity, we explain it in the case when $A_{0}$ and $A_{N}$ are semisimple.

Consider the following linear $q$-difference system:

$$
Y(q x)=A(x) Y(x)
$$

where $A(x)=A_{N} x^{N}+\cdots+A_{1} x+A_{0}$ is an $m \times m$ matrix whose entries are polynomials in $x$. We assume that, for any $a \in \mathbb{C}, A(a) \neq O$. Let the eigenvalues of $A_{0}$ be $\theta_{j}(j=1, \ldots, k)$, and let their multiplicities be $m_{j}(j=1, \ldots, k)$. Similarly, let the eigenvalues of $A_{N}$ be $\kappa_{j}(j=1, \ldots, \ell)$, and let their multiplicities be $n_{j}(j=1, \ldots, \ell)$ :

$$
A_{0} \sim \theta_{1} I_{m_{1}} \oplus \cdots \oplus \theta_{k} I_{m_{k}}, \quad A_{N} \sim \kappa_{1} I_{n_{1}} \oplus \cdots \oplus \kappa_{\ell} I_{n_{\ell}}
$$

Then we define partitions $S_{0}$ and $S_{\infty}$ of $m$ as

$$
S_{0}=m_{1}, \ldots, m_{k}, \quad S_{\infty}=n_{1}, \ldots, n_{\ell}
$$


Let $Z_{A}$ be the set of the zeros of $\operatorname{det} A(x)$ :

$$
Z_{A}=\{a \in \mathbb{C} \mid \operatorname{det} A(a)=0\}=\left\{\alpha_{1}, \ldots, \alpha_{p}\right\} .
$$

We denote by $d_{i}(i=1, \ldots, m)$ the elementary divisors of $A(x)$. Here we assume that $d_{i+1} \mid d_{i}$ (which is opposite to the usual convention). For any $\alpha_{i} \in Z_{A}$, we denote by $\tilde{n}_{k}^{i}$ the order of $\alpha_{i}$ in $d_{k}$. Let $\left\{n_{j}^{i}\right\}_{j}$ be the partition conjugate to $\left\{\tilde{n}_{k}^{i}\right\}_{k}$. Then we define $S_{\text {div }}$ by

$$
S_{\mathrm{div}}=n_{1}^{1} \ldots n_{k_{1}}^{1}, \ldots, n_{1}^{p} \ldots n_{k_{p}}^{p}
$$

So far, we have seen the spectral type of both linear differential equations and linear $q$-difference equations. Concerning the relationship between the two kinds of spectral types, we propose the following conjecture.

Conjecture 3.3. Consider an $m \times m$ system of Fuchsian differential equations:

$$
\frac{d Y}{d x}=\sum_{j=0}^{n} \frac{\mathcal{A}_{j}}{x-u_{j}} Y, \quad u_{0}:=0 .
$$

Let $\mathcal{P}_{j}=m_{1}^{j}, \ldots, m_{\ell_{j}}^{j}(j=0, \ldots, n, \infty)$ be the partition of $m$ corresponding to $\mathcal{A}_{j}$. Thus its spectral type reads $\mathcal{P}_{0} ; \ldots ; \mathcal{P}_{n} ; \mathcal{P}_{\infty}$.

For the equation (3.22), consider an $m \times m$ linear $q$-difference system

$$
Y(q x)=A(x) Y(x), \quad A(x)=A_{n} x^{n}+\cdots+A_{0}
$$

with the following spectral type:

$$
S_{0}=\mathcal{P}_{0}, \quad S_{\infty}=\mathcal{P}_{\infty}, \quad S_{\mathrm{div}}=m_{1}^{1}, \ldots, m_{\ell_{1}}^{1}, \ldots, m_{1}^{n}, \ldots, m_{\ell_{n}}^{n} .
$$

Changing the dependent variable as $Z(x)=f(x) Y(x)$ with a suitable scalar function $f(x)$, we have

$$
\frac{Z(x)-Z(q x)}{(1-q) x}=\frac{1}{(1-q) x}\left\{I_{m}-\frac{f(q x)}{f(x)} A(x)\right\} Z(x) .
$$

Then, by taking a continuous limit $q \rightarrow 1$ such that

$$
\alpha_{j}^{k}\left(j=1, \ldots, \ell_{k}\right) \rightarrow u_{k} \quad(q \rightarrow 1)
$$

where $\alpha_{j}^{k}$ is the zero of $\operatorname{det} A(x)$ corresponding to $m_{j}^{k}$, we can let the equation (3.24) tend to (3.22).

If the conjecture is true, it is expected that the connection-preserving deformation equation of (3.23) gives a $q$-analogue of the isomonodromic deformation equation of (3.22).

\section{Derivation of a $q$-analogue of the matrix $P_{\mathrm{VI}}$}

In this section we derive a $q$-analogue of the matrix $P_{\mathrm{VI}}$. We consider a linear $q$-difference system of spectral type $(m, m ; m, m-1,1 ; m, m, m, m)$, which by Conjecture 3.3 is expected to give a $q$-analogue of the linear system (2.5) associated with the matrix $P_{\mathrm{VI}}$. 


\subsection{Parametrization of linear systems}

First we parametrize linear $q$-difference systems of spectral type $(m, m ; m, m-1,1 ; m, m, m, m)$. Consider a linear $q$-difference system of the following form:

$$
Y(q x)=A(x) Y(x), \quad A(x)=A_{2} x^{2}+A_{1} x+A_{0}, \quad A_{j} \in M_{2 m}(\mathbb{C}),
$$

where

$$
A_{2}=\left(\begin{array}{cc}
\kappa_{1} I_{m} & O \\
O & K
\end{array}\right), \quad K=\operatorname{diag}(\overbrace{\kappa_{2}, \ldots, \kappa_{2}}^{m-1}, \kappa_{3}), \quad A_{0}=G_{0}\left(\begin{array}{cc}
\theta_{1} t I_{m} & O \\
O & \theta_{2} t I_{m}
\end{array}\right) G_{0}^{-1} .
$$

We partition the matrix $A_{1}$ as follows:

$$
A_{1}=\left(\begin{array}{ll}
A_{11} & A_{12} \\
A_{21} & A_{22}
\end{array}\right), \quad A_{i j} \in M_{m}(\mathbb{C}) .
$$

Since $S_{\mathrm{div}}=m, m, m, m$, the Smith normal form of the polynomial matrix $A(x)$ is the following (note that we follow the usual convention here):

$$
\left(\begin{array}{cc}
I_{m} & O \\
O & \prod_{i=1}^{4}\left(x-\alpha_{i}\right) I_{m}
\end{array}\right)
$$

We assume that $\alpha_{j}$ 's depend on $t$ as follows:

$$
\alpha_{j}=\left\{\begin{array}{rr}
a_{j} t & (j=1,2) \\
a_{j} & (j=3,4)
\end{array}\right.
$$

We also assume $q \alpha_{i} \neq \alpha_{j}(i \neq j)$. The parameter $t$ will play the role of a deformation parameter later, then the parameters $\theta_{j}, \kappa_{j}$, and $a_{j}$ 's are independent of $t$. Since

$$
\operatorname{det} A(x)=\kappa_{1}^{m} \kappa_{2}^{m-1} \kappa_{3} \prod_{i=1}^{4}\left(x-\alpha_{i}\right)^{m}
$$

we have

$$
\kappa_{1}^{m} \kappa_{2}^{m-1} \kappa_{3} \prod_{i=1}^{4} a_{i}^{m}=\theta_{1}^{m} \theta_{2}^{m} .
$$

By the assumption above, the matrix $A_{0}$ can be written as follows:

$$
A_{0}=\theta_{2} t I_{2 m}+\left(\begin{array}{c}
I_{m} \\
B_{1}
\end{array}\right)\left(\left(\begin{array}{ll}
\left.\theta_{1}-\theta_{2}\right) t I_{m}-C_{1} B_{1} & C_{1}
\end{array}\right) .\right.
$$

Thus the matrix $A(x)$ reads

$$
A(x)=\left(\begin{array}{cc}
\kappa_{1} I_{m} x^{2}+A_{11} x+\left(\theta_{1} t I_{m}-C_{1} B_{1}\right) & A_{12} x+C_{1} \\
A_{21} x+B_{1}\left\{\left(\theta_{1}-\theta_{2}\right) t I_{m}-C_{1} B_{1}\right\} & K x^{2}+A_{22} x+\left(\theta_{2} t I_{m}+B_{1} C_{1}\right)
\end{array}\right) .
$$


In the same way as in [6], we introduce new variables $F, G_{1}, G_{2}$, and $W$ by

$$
A(x)=\left(\begin{array}{cc}
W K\left\{\kappa_{1}\left(x I_{m}-F\right)\left(x I_{m}-\boldsymbol{\alpha}\right)+\kappa_{1} G_{1}\right\} K^{-1} W^{-1} & W K\left(x I_{m}-F\right) \\
A_{21} x+B_{1}\left\{\left(\theta_{1}-\theta_{2}\right) t I_{m}-C_{1} B_{1}\right\} & K\left(x I_{m}-\boldsymbol{\beta}\right)\left(x I_{m}-F\right)+K G_{2}
\end{array}\right) .
$$

Below we show that the matrix $A(x)$ can be expressed using $F, G_{1}, G_{2}$, and $W$ (indeed, $G_{1}$ and $G_{2}$ are written by $F$ and another matrix $G$ ). First, we immediately have

$$
\begin{aligned}
& C_{1}=-W K F, \quad A_{12}=W K, \\
& A_{11}=-\kappa_{1} W K(F+\boldsymbol{\alpha}) K^{-1} W^{-1}, \quad A_{22}=-K(F+\boldsymbol{\beta}), \\
& \theta_{1} t I_{m}-C_{1} B_{1}=\kappa_{1} W K\left(F \boldsymbol{\alpha}+G_{1}\right) K^{-1} W^{-1}, \quad \theta_{2} t I_{m}+B_{1} C_{1}=K\left(\boldsymbol{\beta} F+G_{2}\right) .
\end{aligned}
$$

Let $M_{1}$ and $M_{2}$ be the following polynomial matrices:

$$
\begin{aligned}
& M_{1}=\left(\begin{array}{cc}
I_{m} & O \\
O & G_{1} K^{-1}
\end{array}\right)\left(\begin{array}{cc}
I_{m} & O \\
Z & I_{m}
\end{array}\right)\left(\begin{array}{cc}
\kappa_{1}^{-1} W K G_{1}^{-1} K^{-1} W^{-1} & O \\
O & I_{m}
\end{array}\right), \\
& M_{2}=\left(\begin{array}{cc}
I_{m} & O \\
-\kappa_{1}\left(x I_{m}-\boldsymbol{\alpha}\right) K^{-1} W^{-1} & I_{m}
\end{array}\right)\left(\begin{array}{cc}
I_{m} & -\kappa_{1}^{-1} W K G_{1}^{-1}\left(x I_{m}-F\right) \\
O & I_{m}
\end{array}\right),
\end{aligned}
$$

where $Z$ is the $(2,1)$-block of

$$
-A(x)\left(\begin{array}{cc}
I_{m} & O \\
-\kappa_{1}\left(x I_{m}-\boldsymbol{\alpha}\right) K^{-1} W^{-1} & I_{m}
\end{array}\right) .
$$

Then by direct calculation we have

$$
M_{1} A(x) M_{2}=\left(\begin{array}{cc}
I_{m} & O \\
O & \tilde{A}(x)
\end{array}\right)
$$

with

$$
\tilde{A}(x)=\left(x^{3}+Q_{1} x^{2}+Q_{2} x+Q_{3}\right)\left(x I_{m}-F\right)+G_{1} G_{2} .
$$

Here $Q_{1}, Q_{2}$, and $Q_{3}$ are written as follows:

$$
\begin{aligned}
& Q_{1}=-G_{1}(\boldsymbol{\alpha}+\boldsymbol{\beta}+F) G_{1}^{-1}, \\
& Q_{2}=G_{1}\left(G_{1}+G_{2}-\kappa_{1}^{-1} K^{-1} A_{21} A_{12}+F \boldsymbol{\alpha}+\boldsymbol{\beta} \boldsymbol{\alpha}+\boldsymbol{\beta} F\right) G_{1}^{-1}, \\
& Q_{3}=-G_{1}\left(\boldsymbol{\beta} G_{1}+G_{2} \boldsymbol{\alpha}+\kappa_{1}^{-1} K^{-1}\left(A_{0}\right)_{21} A_{12}+\boldsymbol{\beta} F \boldsymbol{\alpha}\right) G_{1}^{-1},
\end{aligned}
$$

where $\left(A_{0}\right)_{21}$ is the $(2,1)$-block of $A_{0}$. By the assumption, the Smith normal form of $\tilde{A}(x)$ is $\prod_{j=1}^{4}\left(x-\alpha_{j}\right) I_{m}$. Thus we have

$$
\tilde{A}(x)=\prod_{j=1}^{4}\left(x-\alpha_{j}\right) I_{m}
$$


since $\operatorname{deg} \tilde{A}(x)=4$. Equating the coefficient matrices of $x^{j}$ of both sides of (4.22), we have

$$
\begin{aligned}
Q_{1} & =F+\beta_{1}, \\
Q_{2} & =\left(F+\beta_{1}\right) F+\beta_{2}, \\
Q_{3} & =\left(F^{2}+\beta_{1} F+\beta_{2}\right) F+\beta_{3}, \\
G_{1} G_{2} & =\left(F-\alpha_{1} I\right)\left(F-\alpha_{2} I\right)\left(F-\alpha_{3} I\right)\left(F-\alpha_{4} I\right) .
\end{aligned}
$$

Here the auxiliary parameters $\beta_{j}$ 's are defined by

$$
\sum_{j=0}^{4} \beta_{4-j} x^{j}:=\prod_{j=1}^{4}\left(x-\alpha_{j}\right)
$$

In particular, because of (4.26), we can introduce a new variable $G$ by

$$
\begin{aligned}
& G_{1}=q^{-1} \kappa_{1}^{-1}\left(F-\alpha_{1}\right)\left(F-\alpha_{2}\right) G^{-1}, \\
& G_{2}=q \kappa_{1} G\left(F-\alpha_{3}\right)\left(F-\alpha_{4}\right) .
\end{aligned}
$$

By means of the equations (4.23), (4.24), (4.25), and (4.13), we have

$$
\begin{aligned}
B_{1} & =\left(\kappa_{1}^{-1}-K^{-1}\right)^{-1}\left\{F^{-1} G_{1}+G_{2} F^{-1}-t\left(\theta_{1} \kappa_{1}^{-1}+\theta_{2} K^{-1}\right) F^{-1}-F-G_{1}^{-1} F G_{1}-\beta_{1}\right\} K^{-1} W^{-1}, \\
\boldsymbol{\alpha} & =\left(\kappa_{1}-K\right)^{-1}\left\{\left(\theta_{1}+\theta_{2}\right) t F^{-1}-\kappa_{1} F^{-1} G_{1}-K G_{2} F^{-1}+K\left(F+G_{1}^{-1} F G_{1}+\beta_{1}\right)\right\}, \\
\boldsymbol{\beta} & =\left(\kappa_{1}-K\right)^{-1}\left\{-\left(\theta_{1}+\theta_{2}\right) t F^{-1}+\kappa_{1} F^{-1} G_{1}+K G_{2} F^{-1}-\kappa_{1}\left(F+G_{1}^{-1} F G_{1}+\beta_{1}\right)\right\} \\
A_{21} & =\kappa_{1} K\left\{G_{1}+G_{2}+F \boldsymbol{\alpha}+\boldsymbol{\beta} F+\boldsymbol{\beta} \boldsymbol{\alpha}-G_{1}^{-1}\left(F^{2}+\beta_{1} F+\beta_{2}\right) G_{1}\right\} K^{-1} W^{-1},
\end{aligned}
$$

and

$$
F^{-1} G F G^{-1}=\frac{a_{1} a_{2} a_{3} a_{4} \kappa_{1}}{\theta_{1} \theta_{2}} K
$$

The equation (4.34) is a commutation relation between $F$ and $G$.

From the above we obtain a parametrization of linear $q$-difference systems with spectral type $(m, m ; m, m-1,1 ; m, m, m, m)$ :

$A(x)=A(x, t)=\left(\begin{array}{cc}W K\left\{\kappa_{1}\left(x I_{m}-F\right)\left(x I_{m}-\boldsymbol{\alpha}\right)+\kappa_{1} G_{1}\right\} K^{-1} W^{-1} & W K\left(x I_{m}-F\right) \\ \kappa_{1}(\boldsymbol{\gamma} x+\boldsymbol{\delta}) W^{-1} & K\left(x I_{m}-\boldsymbol{\beta}\right)\left(x I_{m}-F\right)+K G_{2}\end{array}\right)$

with (4.34). Here $\boldsymbol{\gamma}$ and $\boldsymbol{\delta}$ are given by

$$
\begin{aligned}
& \boldsymbol{\gamma}=K\left\{G_{1}+G_{2}+F \boldsymbol{\alpha}+\boldsymbol{\beta} F+\boldsymbol{\beta} \boldsymbol{\alpha}-G_{1}^{-1}\left(F^{2}+\beta_{1} F+\beta_{2}\right) G_{1}\right\} K^{-1}, \\
& \boldsymbol{\delta}=\kappa_{1}^{-1}\left\{t^{2} \theta_{1} \theta_{2} F^{-1}-\kappa_{1} K\left(G_{2}+\boldsymbol{\beta} F\right) F^{-1}\left(G_{1}+F \boldsymbol{\alpha}\right)\right\} K^{-1} .
\end{aligned}
$$




\subsection{The number of accessory parameters}

In the previous subsection, we have seen that the linear $q$-difference systems with spectral type $(m, m ; m, m-1,1 ; m, m, m, m)$ are expressed by $F, G$, and the gauge variable $W$. In this subsection we see that, by the commutation relation (4.34), $F$ and $G$ can be expressed by $2 m$ parameters other than the gauge freedom which comes from $\operatorname{Stab}(K)$. In other words, the number of accessory parameters of the linear system under consideration is $2 m$.

First, using the action of $\operatorname{Stab}(K)$, we can assume that $F$ or $G$ has a particular form. Here we assume that $F$ has the following form (in a similar manner to the proof of Proposition 4.2 in [7]):

$$
F=\left(\begin{array}{cccccc}
0 & 1 & 0 & \ldots & \ldots & 0 \\
0 & 0 & 1 & \ddots & & 0 \\
\vdots & \vdots & \ddots & \ddots & \ddots & \vdots \\
0 & 0 & & \ddots & \ddots & 0 \\
f_{2} & f_{3} & \ldots & & f_{m} & 1 \\
f_{1} & f_{m 2} & f_{m 3} & \ldots & f_{m, m-1} & f_{m m}
\end{array}\right)
$$

Note that the principal $(m-1) \times(m-1)$ submatrix of $F$ obtained by deleting the last row and column is in the form of a companion matrix. Rewrite the commutation relation (4.34) as

$$
G F-\rho F K G=O,
$$

where $\rho=a_{1} a_{2} a_{3} a_{4} \kappa_{1}\left(\theta_{1} \theta_{2}\right)^{-1}$. By the relation (4.7), we have $\operatorname{det}(\rho K)=1$. Regarding (4.39) as a system of linear equations with respect to $G=\left(\boldsymbol{g}_{1}, \ldots, \boldsymbol{g}_{m}\right)$, we further rewrite it as

$$
\Omega_{m} \boldsymbol{g}=\mathbf{0}, \quad \Omega_{m}:={ }^{t} F \otimes I_{m}-I_{m} \otimes(\rho F K),
$$

where

$$
\boldsymbol{g}=\left(\begin{array}{c}
\boldsymbol{g}_{1} \\
\vdots \\
\boldsymbol{g}_{m}
\end{array}\right) .
$$

Now assume that (4.40) has an invertible solution $G$. Then

$$
\left(G^{\oplus m}\right)^{-1} \Omega_{m} G^{\oplus m}={ }^{t} F \otimes I_{m}-I_{m} \otimes F .
$$

By the Cayley-Hamilton theorem, we find that $\operatorname{rank}\left({ }^{t} F \otimes I_{m}-I_{m} \otimes F\right)=m(m-1)$. Thus we have

$$
\operatorname{rank} \Omega_{m}=m(m-1) .
$$

On the other hand, by elementary row operations, $\Omega_{m}$ can be reduced to

$$
\Omega_{m} \rightarrow\left(\begin{array}{ccccc}
O & O & \ldots & O & \Phi_{F}(\tilde{F}) \\
I_{m} & -\tilde{F} & \ldots & \ldots & * \\
& I_{m} & \ddots & & \vdots \\
& & \ddots & \ddots & \vdots \\
& & & I_{m} & f_{m m}-\tilde{F}
\end{array}\right) .
$$


We have written $\rho F K$ as $\tilde{F}$ for simplicity. For an $m \times m$ matrix $M$, we denote the characteristic polynomial of $M$ by $\Phi_{M}(\lambda)$ :

$$
\Phi_{M}(\lambda)=\lambda^{m}-c_{1}(M) \lambda^{m-1}+\cdots+(-1)^{m-k} c_{m-k}(M) \lambda^{k}+\cdots+(-1)^{m} c_{m}(M) .
$$

The condition (4.43) implies $\Phi_{F}(\tilde{F})=O$. Taking the difference of $\Phi_{F}(\tilde{F})=O$ and $\Phi_{\tilde{F}}(\tilde{F})=O$, we have

$$
\begin{aligned}
-\left(c_{1}(F)-c_{1}(\tilde{F})\right) \tilde{F}^{m-1}+\cdots+(-1)^{m-k}\left(c_{m-k}(F)\right. & \left.-c_{m-k}(\tilde{F})\right) \tilde{F}^{k}+\cdots \\
& +(-1)^{m-1}\left(c_{m-1}(F)-c_{m-1}(\tilde{F})\right) \tilde{F}=O
\end{aligned}
$$

(we have used $c_{m}(F)=c_{m}(\tilde{F})$ ). Note that

$$
\left(\tilde{F}^{k}\right)_{1 m}=\left\{\begin{array}{cc}
0 & (k=1, \ldots, m-2), \\
\rho^{m-1} \kappa_{2}^{m-2} \kappa_{3} & (k=m-1) .
\end{array}\right.
$$

Hence the $(1, m)$ entry of (4.46) reads

$$
c_{1}(F)-c_{1}(\tilde{F})=0 .
$$

Multiplying (4.46) by $\tilde{F}$ and again from the $(1, m)$ entry we have

$$
c_{2}(F)-c_{2}(\tilde{F})=0 .
$$

By repeating this procedure, we finally have

$$
c_{k}(F)-c_{k}(\tilde{F})=0 \quad(k=1, \ldots, m-1) .
$$

When we regard (4.50) as equations with respect to $f_{m 2}, \ldots, f_{m m}$, they are uniquely expressed by $f_{1}, \ldots, f_{m}$. Conversely, it is obvious that (4.50) implies (4.43). Under the condition (4.43), the equation (4.40) has $m$ independent solutions and thus its general solution contains $m$ arbitrary constants, say $g_{1}, \ldots, g_{m}$. Therefore $F$ and $G$ are expressed by these $2 m$ accessory parameters $f_{i}$ 's and $g_{i}$ 's $(i=1, \ldots, m)$.

\subsection{Rationality of $B(x, t)$}

Let us consider the connection-preserving deformation of (4.1). Let $\sigma_{t}$ be the $q$-shift operator with respect to $t$, namely, $\sigma_{t}: t \mapsto q t$. For a (matrix valued) function $M=M(t)$, we often denote $\sigma_{t}(M)$ by $\bar{M}$.

By virtue of Proposition 3.1, there exist the following local solutions of (4.1)

$$
\begin{aligned}
& Y_{0}(x, t)=G_{0} \hat{Y}_{0}(x, t) x^{D_{0}}, \quad D_{0}=\frac{1}{\log q} \operatorname{diag}(\overbrace{\log \left(\theta_{1} t\right), \ldots, \log \left(\theta_{1} t\right)}^{m}, \overbrace{\log \left(\theta_{2} t\right), \ldots, \log \left(\theta_{2} t\right)}^{m}), \\
& Y_{\infty}(x, t)=q^{u(u-1)} \hat{Y}_{\infty}(x, t) x^{D_{\infty}}, \quad D_{\infty}=\frac{1}{\log q} \operatorname{diag}(\overbrace{\log \kappa_{1}, \ldots, \log \kappa_{1}}^{m}, \overbrace{\log \kappa_{2}, \ldots, \log \kappa_{2}}^{m}, \log \kappa_{3}) .
\end{aligned}
$$


We note that

$$
\sigma_{t}\left(D_{0}\right)=D_{0}+I_{2 m}, \quad \sigma_{t}\left(D_{\infty}\right)=D_{\infty}
$$

The corresponding connection matrix $P(x, t)$ is defined by $P(x, t)=Y_{0}(x, t)^{-1} Y_{\infty}(x, t)$.

Now assume that $\sigma_{t}(P(x, t))=P(x, t)$. Then by Proposition 3.2 , we have

$$
\left(\sigma_{t} Y_{\infty}\right) Y_{\infty}^{-1}=\left(\sigma_{t} Y_{0}\right) Y_{0}^{-1}
$$

We denote this matrix by $B(x, t)$ :

$$
B(x, t):=\left(\sigma_{t} Y_{\infty}\right) Y_{\infty}^{-1}=\left(\sigma_{t} Y_{0}\right) Y_{0}^{-1} .
$$

Proposition 4.1. The matrix $B(x, t)$ is rational in $x$. More specifically, it has the following form:

$$
B(x, t)=\frac{x\left(x^{2 m-1}+\tilde{B}_{2 m-2} x^{2 m-2}+\cdots+\tilde{B}_{0}\right)}{\left(x-q a_{1} t\right)^{m}\left(x-q a_{2} t\right)^{m}},
$$

where $\tilde{B}_{j}$ 's are $2 m \times 2 m$ matrices.

Proof. Substituting (4.51) and (4.52) into (4.55), we see that $B(x, t)$ is equal to

$$
\hat{Y}_{\infty}(x, q t) \hat{Y}_{\infty}(x, t)^{-1}=\bar{G}_{0} \hat{Y}_{0}(x, q t) x^{\bar{D}_{0}} x^{-D_{0}} \hat{Y}_{0}(x, t)^{-1} G_{0}^{-1} .
$$

Therefore, as a meromorphic function of $x$ on $\mathbb{P}^{1} \backslash\{0, \infty\}$, we find that $B(x, t)$ has the following properties:

- The poles common to both sides are $x=q a_{1} t, q a_{2} t$, and their orders are at most $m$.

- $(\mathrm{LHS})=\left(I+O\left(x^{-1}\right)\right)\left(I+O\left(x^{-1}\right)\right)=I+O\left(x^{-1}\right) \quad(x \rightarrow \infty)$.

- $(\mathrm{RHS})=x \bar{G}_{0}(I+O(x))(I+O(x)) G_{0}^{-1}=O(x) \quad(x \rightarrow 0)$.

These conditions imply that $B(x, t)$ is a meromorphic function on $\mathbb{P}^{1}$, namely, a rational function in $x$ of the following form:

$$
B(x, t)=\frac{x \tilde{B}(x, t)}{\left(x-q a_{1} t\right)^{m}\left(x-q a_{2} t\right)^{m}}, \quad \tilde{B}(x, t)=x^{2 m-1}+\tilde{B}_{2 m-2} x^{2 m-2}+\cdots+\tilde{B}_{0} .
$$

Next we consider the compatibility condition

$$
A(x, q t) B(x, t)=B(q x, t) A(x, t) .
$$

This reads

$$
q^{2 m-1}\left(x-a_{1} t\right)^{m}\left(x-a_{2} t\right)^{m} A(x, q t) \tilde{B}(x, t)=\left(x-q a_{1} t\right)^{m}\left(x-q a_{2} t\right)^{m} \tilde{B}(q x, t) A(x, t) .
$$


It follows that

$$
\begin{aligned}
\exists L, A(x, q t) \tilde{B}(x, t) & =\left(x-q a_{1} t\right)^{m}\left(x-q a_{2} t\right)^{m}\left(A_{2} x+L\right), \\
\exists L^{\prime}, \tilde{B}(q x, t) A(x, t) & =q^{2 m-1}\left(x-a_{1} t\right)^{m}\left(x-a_{2} t\right)^{m}\left(A_{2} x+L^{\prime}\right), \\
L & =L^{\prime} .
\end{aligned}
$$

Conversely, it is easy to see that (4.61), (4.62), and (4.63) imply the compatibility condition (4.59).

The following holds.

\section{Proposition 4.2.}

$$
\left(x-q a_{1} t\right)^{m-1}\left(x-q a_{2} t\right)^{m-1} \mid \tilde{B}(x, t) .
$$

Proof. By the compatibility condition, we have

$$
A(x, q t) \tilde{B}(x, t)=\left(x-q a_{1} t\right)^{m}\left(x-q a_{2} t\right)^{m}\left(A_{2} x+L\right) .
$$

By the relations (4.17) and (4.22), we have

$$
{\overline{M_{1}}}^{-1}\left(\begin{array}{cc}
I_{m} & O \\
O & \prod_{j=1}^{4}\left(x-\overline{\alpha_{j}}\right) I_{m}
\end{array}\right){\overline{M_{2}}}^{-1} \tilde{B}(x, t)=\left(x-q a_{1} t\right)^{m}\left(x-q a_{2} t\right)^{m}\left(A_{2} x+L\right) .
$$

Thus we obtain

$$
\begin{aligned}
\tilde{B}(x, t)= & \left(x-q a_{1} t\right)^{m-1}\left(x-q a_{2} t\right)^{m-1} \\
& \times \overline{M_{2}}\left(\begin{array}{cc}
\left(x-q a_{1} t\right)\left(x-q a_{2} t\right) I_{m} & O \\
O & \left(x-a_{3}\right)^{-1}\left(x-a_{4}\right)^{-1} I_{m}
\end{array}\right) \overline{M_{1}}\left(A_{2} x+L\right) \\
= & \left(x-q a_{1} t\right)^{m-1}\left(x-q a_{2} t\right)^{m-1} \frac{M(x)}{\left(x-a_{3}\right)\left(x-a_{4}\right)},
\end{aligned}
$$

where $M(x)$ is some $2 m \times 2 m$ polynomial matrix in $x$. Since $\left(x-q a_{1} t\right)^{m-1}\left(x-q a_{2} t\right)^{m-1}$ and $\left(x-a_{3}\right)\left(x-a_{4}\right)$ are relatively prime, we find $\left(x-a_{3}\right)\left(x-a_{4}\right) \mid M(x)$. Thus we have

$$
\tilde{B}(x, t)=\left(x-q a_{1} t\right)^{m-1}\left(x-q a_{2} t\right)^{m-1}\left(x+B_{0}\right)
$$

for some matrix $B_{0}$.

From the above discussion, $B(x, t)$ is written as

$$
B(x, t)=\frac{x\left(x I+B_{0}\right)}{\left(x-q a_{1} t\right)\left(x-q a_{2} t\right)} .
$$

Here $B_{0}$ is a $2 m \times 2 m$ matrix independent of $x$. We partition it conformably with $A(x, t)$ :

$$
B_{0}=\left(\begin{array}{ll}
B_{11} & B_{12} \\
B_{21} & B_{22}
\end{array}\right)
$$

where $B_{i j}$ is $m \times m$. $B_{0}$ is determined through the compatibility condition in the next subsection. 


\subsection{Derivation of a $q$-analogue of the matrix $P_{\mathrm{VI}}$}

Consider the matrix $B(x, t)$ given by (4.69). In the same manner as in Section 4.3, we can show that the compatibility condition $A(x, q t) B(x, t)=B(q x, t) A(x, t)$ is equivalent to the following conditions

$$
\begin{aligned}
\exists L, A(x, q t)\left(x+B_{0}\right) & =\left(x-q a_{1} t\right)\left(x-q a_{2} t\right)\left(A_{2} x+L\right), \\
\exists L^{\prime},\left(q x+B_{0}\right) A(x, t) & =q\left(x-a_{1} t\right)\left(x-a_{2} t\right)\left(A_{2} x+L^{\prime}\right), \\
L & =L^{\prime} .
\end{aligned}
$$

First we assume (4.71), (4.72), and (4.73). The conditions (4.71) and (4.72) are respectively equivalent to

$$
\begin{aligned}
A\left(q a_{i} t, q t\right)\left(q a_{i} t I_{2 m}+B_{0}\right)=O & (i=1,2), \\
\left(q a_{i} t I_{2 m}+B_{0}\right) A\left(a_{i} t, t\right) & =O \quad(i=1,2) .
\end{aligned}
$$

The conditions (4.74) and (4.75) imply

$$
\begin{aligned}
& B_{0}=-q a_{i} t I_{2 m}+\left(\begin{array}{c}
I_{m} \\
\Phi^{i}
\end{array}\right) \Lambda_{i}\left(\begin{array}{ll}
\Psi^{i} & I_{m}
\end{array}\right) \quad(i=1,2), \\
& \Phi^{1}=\kappa_{1}\left\{q^{-1} \kappa_{1}^{-1}\left(\bar{F}-q a_{2} t\right) \bar{G}^{-1}-q a_{1} t+\overline{\boldsymbol{\alpha}}\right\} K^{-1} \bar{W}^{-1} \\
& \Phi^{2}=\kappa_{1}\left\{q^{-1} \kappa_{1}^{-1}\left(\bar{F}-q a_{1} t\right) \bar{G}^{-1}-q a_{2} t+\overline{\boldsymbol{\alpha}}\right\} K^{-1} \bar{W}^{-1} \\
& \Psi^{1}=K\left\{-a_{1} t+G_{2}\left(F-a_{1} t\right)^{-1}+\boldsymbol{\beta}\right\} K^{-1} W^{-1}, \\
& \Psi^{2}=K\left\{-a_{2} t+G_{2}\left(F-a_{2} t\right)^{-1}+\boldsymbol{\beta}\right\} K^{-1} W^{-1} .
\end{aligned}
$$

The column vectors of

$$
\left(\begin{array}{c}
I_{m} \\
\Phi^{i}
\end{array}\right)
$$

form a basis of the kernel of left multiplication by $A\left(q a_{i} t, q t\right)$, and the row vectors of

$$
\left(\begin{array}{ll}
\Psi^{i} & I_{m}
\end{array}\right)
$$

form a basis of the kernel of right multiplication by $A\left(a_{i} t, t\right) . \quad \Lambda_{1}$ and $\Lambda_{2}$ are $m \times m$ matrices. Comparing the $(1,2)$-block of

$$
B_{0}=-q a_{1} t I_{2 m}+\left(\begin{array}{l}
I_{m} \\
\Phi^{1}
\end{array}\right) \Lambda_{1}\left(\begin{array}{ll}
\Psi^{1} & I_{m}
\end{array}\right)=-q a_{2} t I_{2 m}+\left(\begin{array}{l}
I_{m} \\
\Phi^{2}
\end{array}\right) \Lambda_{2}\left(\begin{array}{ll}
\Psi^{2} & I_{m}
\end{array}\right),
$$

we have $\Lambda_{1}=\Lambda_{2}=B_{12}$.

On the other hand, by comparing the coefficients of $x^{2}$ of both sides of (4.71), we obtain

$$
A_{2} B_{0}+\overline{A_{1}}=L-q\left(a_{1}+a_{2}\right) t A_{2} .
$$

Also, from the coefficients of $x^{2}$ of (4.72) we have

$$
q A_{1}+B_{0} A_{2}=q L^{\prime}-q\left(a_{1}+a_{2}\right) t A_{2} .
$$


Eliminating $L=L^{\prime}$ from (4.84) and (4.85), we have

$$
\left(q A_{2}\right) B_{0}-B_{0} A_{2}=q\left(A_{1}-\overline{A_{1}}\right)+q(1-q)\left(a_{1}+a_{2}\right) t A_{2} .
$$

From the relation (4.86) we obtain

$$
\begin{aligned}
& B_{11}=\frac{q}{q-1}\left\{\bar{W} K(\overline{\boldsymbol{\alpha}}+\bar{F}) K^{-1} \bar{W}^{-1}-W K(\boldsymbol{\alpha}+F) K^{-1} W^{-1}\right\}-q\left(a_{1}+a_{2}\right) t I_{m}, \\
& B_{12}=q(W-\bar{W}) K\left(q \kappa_{1}-K\right)^{-1} .
\end{aligned}
$$

Comparing the (2,2)-block of (4.83), we have

$$
\left(\Phi^{1}-\Phi^{2}\right) B_{12}=q\left(a_{1}-a_{2}\right) t .
$$

Substituting (4.88) into the above, we obtain

$$
W^{-1} \bar{W}=q \kappa_{1}\left(\bar{G}-K^{-1}\right)^{-1}\left(\bar{G}-\frac{1}{q \kappa_{1}}\right) K^{-1} .
$$

Comparing the (1,1)-block of (4.83), we have

$$
B_{12}\left(\Psi^{1}-\Psi^{2}\right)=q\left(a_{1}-a_{2}\right) t .
$$

Similarly, we get

$$
W^{-1} \bar{W}=K\left(q \kappa_{1}-K\right)^{-1}\left\{-K G_{2}+q \kappa_{1}\left(F-a_{1} t\right)\left(F-a_{2} t\right)\right\}\left\{\left(F-a_{1} t\right)\left(F-a_{2} t\right)-G_{2}\right\}^{-1} K^{-1}\left(q \kappa_{1}-K\right) K^{-1} .
$$

Equating the right-hand sides of (4.90) and (4.92), we obtain

$$
\bar{G} K G=\frac{1}{q \kappa_{1}}\left(F-a_{1} t\right)\left(F-a_{2} t\right)\left(F-a_{3}\right)^{-1}\left(F-a_{4}\right)^{-1} .
$$

Comparing the $(2,1)$-block of (4.83), we have

$$
\Phi^{1} B_{12} \Psi^{1}=\Phi^{2} B_{12} \Psi^{2} .
$$

On the other hand, using the relations (4.89) and (4.91), we have

$$
\begin{aligned}
& \Phi^{1} B_{12} \Psi^{1}=\Phi^{1}\left(q\left(a_{1}-a_{2}\right) t+B_{12} \Psi^{2}\right)=q\left(a_{1}-a_{2}\right) t \Phi^{1}+\left(q\left(a_{1}-a_{2}\right) t+\Phi^{2} B_{12}\right) \Psi^{2} \\
& =q\left(a_{1}-a_{2}\right) t\left(\Phi^{1}+\Psi^{2}\right)+\Phi^{2} B_{12} \Psi^{2} .
\end{aligned}
$$

Thus (4.94) implies

$$
\Phi^{1}+\Psi^{2}=O
$$

It follows that

$$
\overline{\boldsymbol{\alpha}}=-q^{-1} \kappa_{1}^{-1}\left(\bar{F}-q a_{2} t\right) \bar{G}^{-1}+q a_{1} t-\kappa_{1}^{-1} \Psi^{2} \bar{W} K .
$$

By the equation (4.87) and $B_{11}=-q a_{2} t+B_{12} \Psi^{2}$, we have

$$
\bar{W} K(\overline{\boldsymbol{\alpha}}+\bar{F}) K^{-1} \bar{W}^{-1}=W K(\boldsymbol{\alpha}+F) K^{-1} W^{-1}+(q-1) a_{1} t+\frac{q-1}{q} B_{12} \Psi^{2} .
$$


Eliminating $\overline{\boldsymbol{\alpha}}$ using (4.97) and (4.98), we obtain

$$
\bar{F} K F=\frac{\theta_{1} \theta_{2}}{\kappa_{1} a_{1} a_{2}}\left(\bar{G}-t \frac{a_{1} a_{2}}{\theta_{1}}\right)\left(\bar{G}-t \frac{a_{1} a_{2}}{\theta_{2}}\right)\left(\bar{G}-\frac{1}{q \kappa_{1}}\right)^{-1}(\bar{G}-\rho)^{-1} .
$$

Then $B_{i j}$ 's are determined as follows:

$$
\begin{aligned}
B_{11}= & q W K(I-\bar{G} K)^{-1} \bar{G} K\left[K^{-1} \bar{G}^{-1}\left\{F-\left(a_{1}+a_{2}\right) t\right\}+\boldsymbol{\beta}\right] K^{-1} W^{-1}, \\
B_{12}= & q W K(I-\bar{G} K)^{-1} \bar{G} \\
B_{21}= & q \kappa_{1}\left\{q^{-1} \kappa_{1}^{-1}\left(\bar{F}-q a_{2} t\right) \bar{G}^{-1}-q a_{1} t+\overline{\boldsymbol{\alpha}}\right\}\left(I-q \kappa_{1} \bar{G}\right)^{-1} \\
& \times \bar{G} K\left\{K^{-1} \bar{G}^{-1}\left(F-a_{2} t\right)-a_{1} t+\boldsymbol{\beta}\right\} K^{-1} W^{-1}, \\
B_{22}= & {\left[q^{-1} \kappa_{1}^{-1}\left\{\bar{F}-q\left(a_{1}+a_{2}\right) t\right\} \bar{G}^{-1}+\overline{\boldsymbol{\alpha}}\right] q \kappa_{1} \bar{G}\left(I-q \kappa_{1} \bar{G}\right)^{-1} }
\end{aligned}
$$

Conversely, by direct calculation, we can show that the equations (4.90), (4.93), and (4.99) are also the sufficient condition to the compatibility condition. Thus we have the following

Theorem 4.3. The compatibility condition $A(x, q t) B(x, t)=B(q x, t) A(x, t)$ is equivalent to

$$
\begin{aligned}
\bar{F} K F & =\frac{\theta_{1} \theta_{2}}{\kappa_{1} a_{1} a_{2}}\left(\bar{G}-t \frac{a_{1} a_{2}}{\theta_{1}}\right)\left(\bar{G}-t \frac{a_{1} a_{2}}{\theta_{2}}\right)\left(\bar{G}-\frac{1}{q \kappa_{1}}\right)^{-1}(\bar{G}-\rho)^{-1}, \\
\bar{G} K G & =\frac{1}{q \kappa_{1}}\left(F-a_{1} t\right)\left(F-a_{2} t\right)\left(F-a_{3}\right)^{-1}\left(F-a_{4}\right)^{-1}, \\
W^{-1} \bar{W} & =q \kappa_{1}\left(\bar{G}-K^{-1}\right)^{-1}\left(\bar{G}-\frac{1}{q \kappa_{1}}\right) K^{-1} .
\end{aligned}
$$

We call the equations (4.104) and (4.105) (with (4.34) $) q$-matrix $P_{\mathrm{VI}}$ since they have a continuous limit $q \rightarrow 1$ to the matrix $P_{\mathrm{VI}}$ (see Section 5 ). Note that this system can be regarded as a nonabelian analogue of Jimbo-Sakai's $q-P_{\mathrm{VI}}$, so we also call it matrix $q-P_{\mathrm{VI}}$.

Although this system appears to have eight parameters $\left(\theta_{i}\right.$ 's, $\kappa_{i}$ 's, and $a_{i}$ 's with a single relation), the number of parameters can be reduced to five by rescaling $F, G$, and $t$.

\section{Continuous limit}

As expected, the system (4.104) and (4.105) can be viewed as a $q$-analogue of the matrix $P_{\mathrm{VI}}(2.15)$ and (2.16). That is, taking the limit $q \rightarrow 1$, one can obtain (2.15) and (2.16) from (4.104) and (4.105). In fact, let us define the parameter $\varepsilon$ by $q=e^{-\varepsilon}$. We set

$$
\theta_{i}=e^{-\varepsilon \sigma_{i}}(i=1,2), \quad a_{i}=e^{\varepsilon \zeta_{i}}(i=1, \ldots, 4), \quad \kappa_{i}=e^{\varepsilon \mu_{i}}(i=1,2,3)
$$

Note that the zeros $\alpha_{1}, \alpha_{2}$ of $A(x, t)$ tend to $t$ and $\alpha_{3}, \alpha_{4}$ tend to 1 when $\varepsilon$ goes to 0 . Moreover, we introduce new dependent variables $Q$ and $P$, which are related to $F$ and $G$ by

$$
F=\tilde{Q}, \quad G=e^{\varepsilon\left(\zeta_{2}+\zeta_{4}+\sigma_{2}\right)} e^{\varepsilon(\tilde{Q} \tilde{P}+I)}\left(\tilde{Q}-e^{\varepsilon \zeta_{1}} t I\right)\left(\tilde{Q}-e^{\varepsilon \zeta_{4}} I\right)^{-1}
$$


and

$$
\begin{aligned}
& \tilde{Q}=(Q-I)^{-1}(Q-t I), \\
& \tilde{P}=\frac{1}{t-1}(Q-I)\left\{P(Q-I)+\zeta_{1}+\zeta_{4}+\mu_{1}+\sigma_{1}-\left(\zeta_{1}-\zeta_{2}\right) Q^{-1}+\left(\sigma_{1}-\sigma_{2}\right)(t-1)(Q-t I)^{-1}\right\} .
\end{aligned}
$$

Then, by taking a limit $\varepsilon \rightarrow 0$, we find that $Q, P$ satisfy the following equations:

$$
\begin{aligned}
t(t & -1) \frac{d Q}{d t} \\
= & (Q-t) P Q(Q-1)+Q(Q-1) P(Q-t) \\
& +\left(\sigma_{1}-\sigma_{2}+1\right) Q(Q-1)+\left(\zeta+2 \mu_{1}+\sigma_{1}+\sigma_{2}-1\right) Q(Q-t)+\left(\zeta_{1}-\zeta_{2}\right)(Q-1)(Q-t) \\
t( & -1) \frac{d P}{d t} \\
= & -(Q-1) P(Q-t) P-P(Q-t) P Q-P Q(Q-1) P \\
& -\left[\left(\sigma_{1}-\sigma_{2}+1\right)\{P(Q-1)+Q P\}+\left(\zeta+2 \mu_{1}+\sigma_{1}+\sigma_{2}-1\right)\{P(Q-t)+Q P\}+\left(\zeta_{1}-\zeta_{2}\right)\{P(Q-t)+(Q-1) P\}\right] \\
& -\left(\zeta_{1}+\zeta_{3}+\sigma_{1}+\mu_{1}\right)\left(\zeta_{1}+\zeta_{4}+\sigma_{1}+\mu_{1}\right)
\end{aligned}
$$

where $\zeta:=\zeta_{1}+\cdots+\zeta_{4}$. These equations coincide with (2.15) and (2.16) with the following correspondence of the parameters:

$$
\sigma_{1}-\sigma_{2}=\theta^{0}, \zeta_{3}-\zeta_{4}=\theta^{1}, \zeta_{1}-\zeta_{2}=\theta^{t}, \mu_{i}+\zeta_{2}+\zeta_{4}+\sigma_{2}=\theta_{i}^{\infty}(i=1,2,3) .
$$

Expanding (4.34) with respect to $\varepsilon$ and taking the coefficient of $\varepsilon^{1}$, we have a commutation relation between $P$ and $Q$ :

$$
[P, Q]=\left(\zeta_{1}+\cdots+\zeta_{4}+\sigma_{1}+\sigma_{2}+\mu_{1}\right) I_{m}+M, \quad M=\operatorname{diag}(\overbrace{\mu_{2}, \ldots, \mu_{2}}^{m-1}, \mu_{3}) .
$$

The linear system (4.1) also admit the continuous limit in a similar way. To see this, we first change the dependent variable $Y$ to $Z(x)=f(x) Y(x)$, where $f(x)$ is a solution of the following $q$-difference equation

$$
f(q x)=\frac{f(x)}{(x-1)(x-t)}
$$

For example, we can take

$$
f(x)=(x ; q)_{\infty}(x / t ; q)_{\infty} \frac{\vartheta_{q}(x)}{\vartheta_{q}(x / t)},
$$

where

$$
(a ; q)_{\infty}=\lim _{n \rightarrow \infty} \prod_{j=0}^{n-1}\left(1-a q^{j}\right), \quad \vartheta_{q}(x)=\prod_{n=0}^{\infty}\left(1-q^{n+1}\right)\left(1+x q^{n}\right)\left(1+x^{-1} q^{n+1}\right) .
$$

Then we have

$$
\frac{Z(x)-Z(q x)}{(1-q) x}=\frac{1}{\varepsilon x(1+O(\varepsilon))}\left\{I_{2 m}-\frac{1}{(x-1)(x-t)} A(x)\right\} Z(x) .
$$


Put $W=\varepsilon U^{-1}(Q-I)$. Define matrices $\mathrm{A}_{0}, \mathrm{~A}_{1}$, and $\mathrm{A}_{t}$ by

$$
\lim _{\varepsilon \rightarrow 0} \frac{1}{\varepsilon x}\left\{I_{2 m}-\frac{1}{(x-1)(x-t)} A(x)\right\}=\frac{\mathrm{A}_{0}}{x}+\frac{\mathrm{A}_{1}}{x-1}+\frac{\mathrm{A}_{t}}{x-t} .
$$

Then the matrices $A_{0}, A_{1}, A_{t}$ (almost) coincide with (2.9). More precisely, we have

$$
\mathrm{A}_{0}-\sigma_{2} I_{2 m}=\mathcal{A}_{0}, \quad \mathrm{~A}_{1}-\zeta_{4} I_{2 m}=\mathcal{A}_{1}, \quad \mathrm{~A}_{t}-\zeta_{2} I_{2 m}=\mathcal{A}_{t} .
$$

Thus the resulting system of linear differential equations

$$
\frac{d Z}{d x}=\left(\frac{\mathrm{A}_{0}}{x}+\frac{\mathrm{A}_{1}}{x-1}+\frac{\mathrm{A}_{t}}{x-t}\right) Z
$$

is of spectral type $m, m ; m, m ; m, m ; m, m-1,1$. In this case, Conjecture 3.3 holds.

\section{References}

[1] P. Boalch, Simply-laced isomonodromy systems, Publ. Math. Inst. Hautes Études Sci. 116, No. 1 (2012), 1-68.

[2] R. D. Carmichael, The general theory of linear q-difference equations, Amer. J. Math. 34, No. 2 (1912), 147-168.

[3] K. Fuji and T. Suzuki, Drinfeld-Sokolov hierarchies of type $A$ and fourth order Painlevé systems, Funkcial. Ekvac. 53 (2010), 143-167.

[4] R. Garnier, Sur des équations différentielles du troisième ordre dont l'intégrale générale est uniforme et sur une classe d'équations nouvelles d'ordre supérieur dont l'intégrale générale a ses points critiques fixes, Ann. Sci. Éc. Norm. Supér. 29 (1912), 1-126.

[5] K. Hiroe, H. Kawakami, A. Nakamura, and H. Sakai, 4-dimensional Painlevé-type equations, MSJ Memoirs 37 (2018).

[6] M. Jimbo and H. Sakai, A q-analog of the sixth Painlevé equation, Lett. Math. Phys. 38 (1996), $145-154$.

[7] H. Kawakami, Matrix Painlevé systems, J. Math. Phys. 56 (2015), doi.org/10.1063/1.4914369.

[8] H. Kawakami, Four-dimensional Painlevé-type equations associated with ramified linear equations III: Garnier systems and FS systems, SIGMA 13 (2017), 096, 50 pages.

[9] H. Kawakami, Four-dimensional Painlevé-type equations associated with ramified linear equations II: Sasano systems, Journal of Integrable Systems, Volume 3, Issue 1 (2018), xyy013.

[10] H. Kawakami, Four-dimensional Painlevé-type equations associated with ramified linear equations I: Matrix Painlevé systems, to appear in Funkcial. Ekvac., arXiv:1608.03927. 
[11] T. Masuda, A q-analogue of the higher order Painlevé type equations with the affine Weyl group symmetry of type D, Funkcial. Ekvac. 58 (2015), 405-430.

[12] T. Oshima, Fractional calculus of Weyl algebra and Fuchsian differential equations, MSJ Memoirs 28 (2012).

[13] H. Sakai, Rational surfaces associated with affine root systems and geometry of the Painlevé equations, Comm. Math. Phys. 220 (2001), 165-229.

[14] H. Sakai, A q-analog of the Garnier system, Funkcial. Ekvac. 48 (2005), 273-297.

[15] H. Sakai and M. Yamaguchi, Spectral types of linear $q$-difference equations and $q$-analog of middle convolution, Int. Math. Res. Not., Volume 2017, Issue 7 (2017), 1975-2013.

[16] Y. Sasano, Coupled Painleve VI systems in dimension four with affine Weyl group symmetry of type $D_{6}^{(1)}$. II, RIMS Kôkyûroku Bessatsu B5 (2008), 137-152.

[17] T. Suzuki, A $q$-analogue of the Drinfeld-Sokolov hierarchy of type $A$ and $q$-Painlevé system, AMS Contemp. Math. 651 (2015), 25-38.

[18] T. Tsuda, On an Integrable System of $q$-Difference Equations Satisfied by the Universal Characters: Its Lax Formalism and an Application to q-Painlevé Equations, Comm. Math. Phys. 293 (2010), 347-359.

[19] T. Tsuda, UC hierarchy and monodromy preserving deformation, J. Reine Angew. Math. 690 (2014), 1-34. 